\title{
Doubly magic nuclei from lattice QCD forces at $M_{\mathrm{PS}}=469 \mathrm{MeV} / c^{2}$
}

\author{
C. McIlroy, ${ }^{1, *}$ C. Barbieri, ${ }^{1, \dagger}$ T. Inoue, ${ }^{2,3}$ T. Doi,,${ }^{3,4}$ and T. Hatsuda ${ }^{3,4}$ \\ ${ }^{1}$ Department of Physics, University of Surrey, Guildford GU2 7XH, United Kingdom \\ ${ }^{2}$ Nihon University, College of Bioresource Sciences, Kanagawa 252-0880, Japan \\ ${ }^{3}$ Theoretical Research Division, Nishina Center, RIKEN, Wako 351-0198, Japan \\ ${ }^{4}$ iTHEMS Program and iTHES Research Group, RIKEN, Wako 351-0198, Japan
}

(Received 23 December 2016; revised manuscript received 31 December 2017; published 20 February 2018)

\begin{abstract}
We perform $a b$ initio self-consistent Green's function calculations of the closed shell nuclei ${ }^{4} \mathrm{He},{ }^{16} \mathrm{O}$, and ${ }^{40} \mathrm{Ca}$, based on two-nucleon potentials derived from lattice QCD simulations, in the flavor SU(3) limit and at the pseudoscalar meson mass of $469 \mathrm{MeV} / c^{2}$. The nucleon-nucleon interaction is obtained using the hadronsto-atomic-nuclei-from-lattice (HAL) QCD method, and its short-distance repulsion is treated by means of ladder resummations outside the model space. Our results show that this approach diagonalizes ultraviolet degrees of freedom correctly. Therefore, ground-state energies can be obtained from infrared extrapolations even for the relatively hard potentials of HAL QCD. Comparing to previous Brueckner Hartree-Fock calculations, the total binding energies are sensibly improved by the full account of many-body correlations. The results suggest an interesting possible behavior in which nuclei are unbound at very large pion masses and islands of stability appear at first around the traditional doubly magic numbers when the pion mass is lowered toward its physical value. The calculated one-nucleon spectral distributions are qualitatively close to those of real nuclei even for the pseudoscalar meson mass considered here.
\end{abstract}

DOI: 10.1103/PhysRevC.97.021303

Introduction. Quantum chromodynamics (QCD) is expected to ultimately explain the structure and the interactions of all hadronic systems, together with small corrections of electroweak origin. At the moment, systematic and nonperturbative calculations of QCD can be carried out only by lattice QCD (LQCD). Indeed, high-precision studies have been shown to be possible, e.g., in single-hadron masses [1]. "Direct" calculations of multibaryon systems have also been attempted on a lattice by several groups [2-6]. However, the typical excitation energy $\Delta E$ for multibaryons is one to two orders of magnitude smaller than $\mathcal{O}\left(\Lambda_{\mathrm{QCD}}\right)$. Accordingly, high statistics data with very large Euclidean times $t \simeq \hbar / \Delta E \approx$ $10-100 \mathrm{fm} / c$ is required. This is still far beyond reach due to exponentially increasing errors in $t$ and $A$ (the atomic number), as demonstrated theoretically and numerically in recent studies [7-9].

In this work, we follow a different route and perform an $a b$ initio study for medium mass atomic nuclei directly based on QCD by taking a two-step strategy. In the first step, we extract the nuclear force from $a b$ initio LQCD calculations with the HAL QCD method. This procedure generates consistent two-, three-, and many-nucleon forces in a systematic way [10-13]. Note that the HAL QCD interactions obtained directly from spatiotemporal hadron-hadron correlations on the lattice are faithful to the scattering phase shifts and binding energies by construction. This is done systematically without the fitting

\footnotetext{
*c.s.mcilroy@ surrey.ac.uk

${ }^{\dagger}$ C.Barbieri@surrey.ac.uk
}

procedures needed by phenomenological potentials. Furthermore, explorative studies of the three-nucleon potential show that this is weaker than the corresponding nucleon-nucleon $(N N)$ force, in agreement with the empirically observed hierarchy of nuclear forces [14]. Thus, the interaction can be applied to larger nuclei. In the second step, we calculate the properties of nuclei with ab initio many-body methods using the LQCD potentials as input. The advantage of the HAL QCD approach is that one can extract the potential dictating all the elastic scattering states below the inelastic threshold from the lattice data for $t \simeq 1 \mathrm{fm} / c$ [15]. This makes the LQCD calculation of potentials affordable with reasonable statistics, together with the help of advanced computational algorithms [16-19]. We can then take the advantage of the recent developments in nuclear many-body theories to calculate various information on nuclei such as binding energies and spectral distributions. Note that a similar two-step strategy has also been taken in Refs. [20,21] where, however, effective field theories have been used to fit the LQCD data.

Past LQCD studies in the flavor SU(3) limit by the HAL QCD Collaboration have led to interactions in both the nucleon and hyperon sectors with masses of the pseudoscalar meson (which corresponds to the pion) as low as $M_{\mathrm{PS}}=469 \mathrm{MeV} / c^{2}$. In these cases, potentials have been obtained for the ${ }^{1} S_{0}$ and the coupled ${ }^{3} S_{1}{ }^{3} D_{1}$ channels [22,23]. Exploratory calculations based on these HAL QCD potentials were performed in the Brueckner Hartree-Fock (BHF) approach [24,25]. This is quantitative enough to give the essential underlying physics for infinite matter but it is less reliable in finite systems. More sophisticated calculations are needed in order to go beyond the mean-field level, which is necessary to properly predict binding 
energies and to describe the truly complex structures of nuclei at low energy. BHF becomes even more questionable for finite nuclei due to assumptions with the unperturbed single-particle spectrum where there is a problem in the choice between a continuous or a gap form, neither of which is completely satisfactory.

$A b$ initio theories for medium mass nuclei have advanced greatly in the past decade and methods such as coupled cluster and self-consistent Green's function (SCGF) are now routinely employed to study the structure of full isotopic chains up to $\mathrm{Ca}$ and $\mathrm{Ni}$ [26-29], with the inclusion of three-nucleon forces [30]. Their use with soft interactions from chiral effective field theory have led to first-principle predictions of experimental total binding energies [31,32] and nuclear radii [33-35] with unprecedented accuracies. Interactions like the HAL QCD potentials pose a bigger challenge for calculations of medium mass isotopes due to their short-distance internucleon repulsion. However, the SCGF method has the advantage that two-nucleon scattering (ladder) diagrams at large momentaneeded to resolve the short-range repulsion — can be dealt with explicitly by solving the Bethe-Goldstone equation (BGE) in the excluded space [36-38]. This route was exploited in the past to study spectral strength distribution but we extend it to binding energies in the present work and find that this is accurate enough to make statements on the performance of the present HAL QCD potentials. Thus, this work is also a step toward advancing many-body approaches that can handle hard interactions for large atomic masses. This is also important since new LQCD calculations at nearly the physical pion mass are currently in progress [39-42].

The BHF study of Ref. [24] showed that the HAL QCD interactions in the SU(3) limit do not bind at very large pion masses except for the lowest available value $M_{\mathrm{PS}}=469$ $\mathrm{MeV} / \mathrm{c}^{2}$. The saturation of nuclear matter in this case is also confirmed by later SCGF calculations [43]. Thus, this is a suitable choice to investigate possible self-bound nuclei at large pion masses. In this work, we will focus on this potential and refer to it as the HAL469 $9_{\mathrm{SU}(3)}$ interaction, or HAL469 for simplicity.

Formalism. We follow closely the approach of Ref. [38] and focus on the single-particle propagator given by $[45,46]$

$$
g_{\alpha \beta}(\omega)=\sum_{n} \frac{\left(\mathcal{X}_{\alpha}^{n}\right)^{*} \mathcal{X}_{\beta}^{n}}{\omega-\varepsilon_{n}^{+}+i \eta}+\sum_{k} \frac{\mathcal{Y}_{\alpha}^{k}\left(\mathcal{Y}_{\beta}^{k}\right)^{*}}{\omega-\varepsilon_{k}^{-}-i \eta},
$$

where $\mathcal{X}_{\alpha}^{n} \equiv\left\langle\Psi_{n}^{A+1}\left|c_{\alpha}^{\dagger}\right| \Psi_{0}^{A}\right\rangle \quad\left(\mathcal{Y}_{\alpha}^{k} \equiv\left\langle\Psi_{k}^{A-1}\left|c_{\alpha}\right| \Psi_{0}^{A}\right\rangle\right)$ are the spectroscopic amplitudes, $\varepsilon_{n}^{+} \equiv E_{n}^{A+1}-E_{0}^{A} \quad\left(\varepsilon_{k}^{-} \equiv E_{0}^{A}-\right.$ $\left.E_{k}^{A-1}\right)$ are their quasiparticle energies, and $c_{\alpha}^{\dagger}\left(c_{\alpha}\right)$ are the second quantization creation (annihilation) operators. In these definitions, $\left|\Psi_{n}^{A+1}\right\rangle$ and $\left|\Psi_{k}^{A-1}\right\rangle$ represent the exact eigenstates of the $(A \pm 1)$-nucleon system, while $E_{n}^{A+1}$ and $E_{k}^{A-1}$ are the corresponding energies. We perform calculations within a spherical harmonic oscillator model space, indicated as $P$, and use Greek indices $\alpha, \beta, \ldots$ to label its basis functions. Within this space, the one-body propagator is obtained by solving the Dyson equation with an irreducible self-energy given by

$$
\Sigma_{\alpha \beta}^{\star}(\omega)=\Sigma_{\alpha \beta}^{(\infty)}+\sum_{i j} \mathbf{D}_{\alpha i}^{\dagger}\left[\frac{1}{\omega-(\mathbf{K}+\mathbf{C}) \pm i \eta}\right]_{i j} \mathbf{D}_{j \beta} .
$$

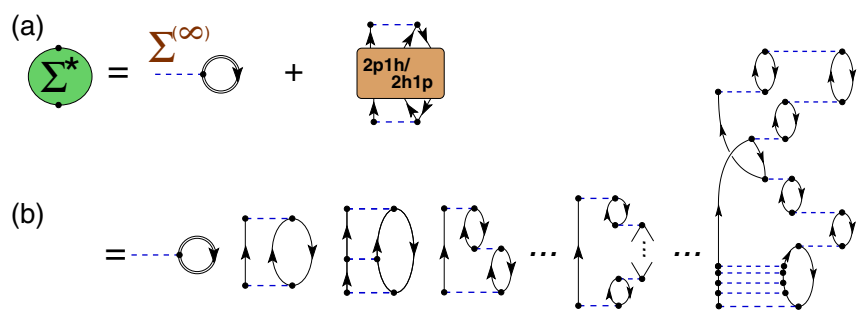

FIG. 1. Diagrammatic content of the ADC(3) approximation. (a) The self-energy splits into a static mean-field part and an energydependent contribution according to Eq. (2). (b) The dynamic contributions are obtained as infinite resummations of ladder $(\mathrm{pp} / \mathrm{hh})$ and ring (ph) diagrams. The ADC(3) approach includes static corrections to the coupling of nucleons to intermediate excitations [matrix $\mathbf{D}$ in Eq. (2)], an example of which is shown by the top portion of the last Goldstone diagram [44].

This expression is the sum of a mean-field (MF) term, $\Sigma^{(\infty)}$, and the contributions from dynamical correlations. The coupling (D) and interactions matrices ( $\mathbf{K}$ and $\mathbf{C}$ ) are computed in the third-order algebraic diagrammatic construction [ADC(3)] approximation that generates nonperturbative all-order summations of $2 \mathrm{p} 1 \mathrm{~h}$ and $2 \mathrm{~h} 1 \mathrm{p}$ configurations, as shown in Fig. 1 $[44,47,48]$. We follow the $s c 0$ approximation of Refs. [38,49], in which $\Sigma^{(\infty)}$ is calculated exactly from the fully dressed propagator $g(\omega)$ while $\mathbf{D}, \mathbf{K}$, and $\mathbf{C}$ are written in terms of a simplified MF reference propagator, $g^{\text {ref }}(\omega)$, which is chosen to best approximate $g(\omega)$ through its first two moments at the Fermi energy (see Ref. [38] for details).

For forces with a sizable short-range repulsion, like the HAL QCD interactions, usual truncations of the oscillator space (of up to 12 shells in this case) are not sufficient and a resummation of ladder diagrams in the excluded Hilbert space, $Q \equiv \mathbf{1}-P$, is required. We do this by solving the BGE in $Q$ according to Refs. [50,51] and add the corresponding diagrams to the MF self-energy, which becomes energy dependent [38]:

$$
\begin{aligned}
\Sigma_{\alpha \beta}^{(\infty)}(\omega) & =\sum_{\gamma \delta} \int \frac{d \omega^{\prime}}{2 \pi i} T_{\alpha \gamma, \beta \delta}^{\mathrm{BGE}}\left(\omega+\omega^{\prime}\right) g_{\gamma \delta}\left(\omega^{\prime}\right) e^{i \omega^{\prime} \eta} \\
& =\sum_{\gamma \delta} \sum_{k} T_{\alpha \gamma, \beta \delta}^{\mathrm{BGE}}\left(\omega+\varepsilon_{k}^{-}\right) \mathcal{Y}_{\delta}^{k}\left(\mathcal{Y}_{\gamma}^{k}\right)^{*}
\end{aligned}
$$

where $T_{\alpha \gamma, \beta \delta}^{\mathrm{BGE}}(\omega)$ are the elements of the scattering $t$ matrix in the excluded space. We then extract a static effective interaction that we use to calculate the $\mathrm{ADC}(3)$ self-energy [the last term of Eq. (2)] within the model space $P$. To do this, we solve the Hartree-Fock (HF) equations with the MF potential of Eq. (3)

$$
\sum_{\beta}\left\{\left\langle\alpha\left|\frac{p^{2}}{2 m_{N}}\right| \beta\right\rangle+\Sigma_{\alpha \beta}^{(\infty)}\left(\omega=\varepsilon_{r}^{\mathrm{HF}}\right)\right\} \psi_{\beta}^{r}=\varepsilon_{r}^{\mathrm{HF}} \psi_{\alpha}^{r},
$$

where latin indices label HF states, and define a static interaction in this HF basis similar to Refs. [38,52]:

$$
V_{r s, p q}=\frac{1}{2}\left[T_{r s, p q}^{\mathrm{BGE}}\left(\varepsilon_{r}^{\mathrm{HF}}+\varepsilon_{s}^{\mathrm{HF}}\right)+T_{r s, p q}^{\mathrm{BGE}}\left(\varepsilon_{p}^{\mathrm{HF}}+\varepsilon_{q}^{\mathrm{HF}}\right)\right] .
$$

The $V_{r s, p q}$ matrix elements are then transformed back to the harmonic oscillator space to be used in the computations. 

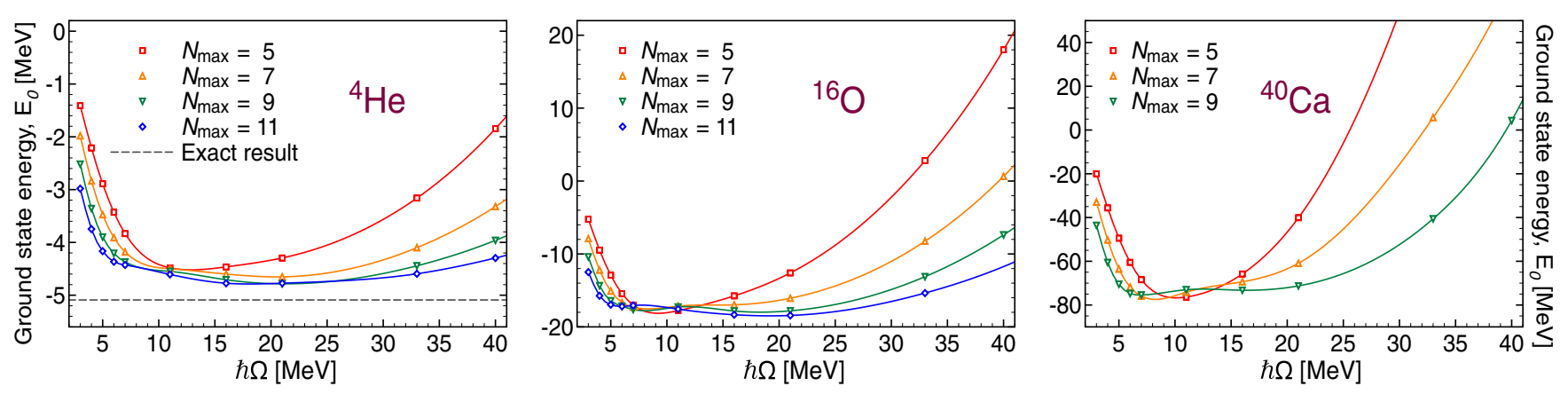

FIG. 2. Ground-state energy of ${ }^{4} \mathrm{He},{ }^{16} \mathrm{O}$, and ${ }^{40} \mathrm{Ca}$ as a function of the harmonic oscillator frequency $\hbar \Omega$ and the model space size $N_{\text {max }}$. Symbols mark the results for the HAL469 potential from full self-consistent calculations in the $T^{\mathrm{BGE}}(\omega)$ plus ADC(3) approach.

Note that the BGE used to generate $T^{\mathrm{BGE}}(\omega)$ resums scattering states where at least one nucleon is outside the whole model space. Hence, it does not depend on the isotope being computed, neither does it suffer from ambiguities with the choice of the single-particle spectrum at the Fermi surface that are encountered with the usual $G$ matrix in BHF calculations. Instead, our approach is very similar to the Bloch-Horowitz method of Ref. [53], of which our $T^{\mathrm{BGE}}(\omega)$ is also a first approximation. The $\mathrm{ADC}(3)$ computation accounts for all types of correlations inside the model space, including all remaining ladder diagrams. This ensures a complete many-body calculation that accounts for short-distance repulsion.

It is well known that short-range repulsion, which is accounted for by Eq. (3), has the double effect of reducing the spectral strength for dominant quasiparticle peaks and of relocating it to large momenta and large quasiparticle energies [29]. Since we cannot currently calculate the location of strength at high momenta, it is not possible to quantify the magnitude of these two effects. However, they contribute to the Koltun sum rule for total binding energy with opposite signs and must cancel to a large extent. Thus, we choose to neglect both contributions and maintain a static $\Sigma^{(\infty)}$ to solve the Dyson equation. This is currently the major approximation in our calculations and its uncertainty is best estimated from the benchmark on ${ }^{4} \mathrm{He}$ below. Resolving this requires a proper extension of the present SCGF formalism and it will be the subject of future work.

Infrared convergence. The one-body propagators of ${ }^{4} \mathrm{He}$, ${ }^{16} \mathrm{O}$, and ${ }^{40} \mathrm{Ca}$ are calculated in spherical harmonic oscillator spaces of different frequencies, $\hbar \Omega$, and increasing sizes up to $N_{\max }=\max \{2 n+\ell\}=11$ (and $N_{\max } \leqslant 9$ for ${ }^{40} \mathrm{Ca}$ ). The scattering matrix $T^{\mathrm{BGE}}(\omega)$ is calculated for each frequency and model space and then used to derive the interactions of Eq. (5). We subtract the kinetic energy of the center of mass according to Ref. [54] and calculate the intrinsic ground-state energy from $g(\omega)$ using the Koltun sum rule. The same lattice simulation setup used to generate the HAL469 interaction gives a nucleon mass of $m_{N}=1161.1 \mathrm{MeV} / c^{2}$ in addition to the pseudoscalar mass of $M_{\mathrm{PS}}=469 \mathrm{MeV} / c^{2}$. Thus, we employ this value of $m_{N}$ in all the kinetic energy terms.

Figure 2 displays the ground-state energies obtained with our $T^{\mathrm{BGE}}(\omega)$ plus $\mathrm{ADC}(3)$ method. As expected, the complete resummation of ladder diagrams outside the model space tames the ultraviolet corrections and results in a rather flat behavior of the total energies for $\hbar \Omega \approx 5-20 \mathrm{MeV}$. Still, there remain some hints of oscillations with respect to $\hbar \Omega$ that could be linked to the $\mathrm{HO}$ truncation and to the neglect of spectral strength at high momenta as explained above.

The effectiveness of the ladder resummation is better recognized by considering the infrared (IR) convergence of the total binding energies, from where one can also extrapolate to a complete set. More et al. [55] established that a harmonic oscillator model space, of frequency $\hbar \Omega$ and truncated to the first $N_{\max }+1$ shells, behaves as a hard wall spherical box of radius

$$
L_{2}=\sqrt{2\left(N_{\max }+3 / 2+2\right)} b,
$$

where $b \equiv \sqrt{\hbar c^{2} / m_{N} \Omega}$ is the oscillator length. Given a soft interaction that is independent of the model space, if the frequency is large enough (i.e., $b$ is small) then ultraviolet (UV) degrees of freedom are converged. In this case, the calculated ground-state energies are expected to converge exponentially when increasing the effective radius $L_{2}$ :

$$
E_{0}^{A}\left[N_{\max }, \hbar \Omega\right]=E_{\infty}+C e^{-2 k_{\infty} L_{2}} .
$$

For the bare HAL469 interaction, if we use the SCGF without ladder sums outside the model space, the extrapolation according to Eq. (7) will fail because the short-distance repulsion requires extremely large $N_{\max }(\gg 20)$ to reach UV convergence, while our many-body space $P$ is limited to $N_{\max }=11$. This is shown by the left panel of Fig. 3. However, our complete calculations use the BGE to resum all missing ladder diagrams within $Q$. Adding these to the two-particle ladders that are generated by the $\mathrm{ADC}(3)$ leads to a complete diagonalization of short-distance degrees of freedom, independently of the choice of $P$. The resulting dependence of the ground-state energy on $L_{2}$ is shown by the middle panel and it now follows the behavior dictated by Eq. (7). Note that single-particle energies are still needed to calculate $T^{\mathrm{BGE}}(\omega)$ but these can be identified with the free particle spectrum in the space $Q$, at large momenta. Accordingly, the flat region in Fig. 3 becomes broader as we increase $N_{\max }$ (that is, when the boundary between the $P$ and $Q$ spaces moves away from the Fermi surface).

The binding energy of ${ }^{4} \mathrm{He}$ for HAL469 was found to be $-5.09 \mathrm{MeV}$ with the exact stochastic variational calculations [56], which we will use to benchmark our approach. The solid 

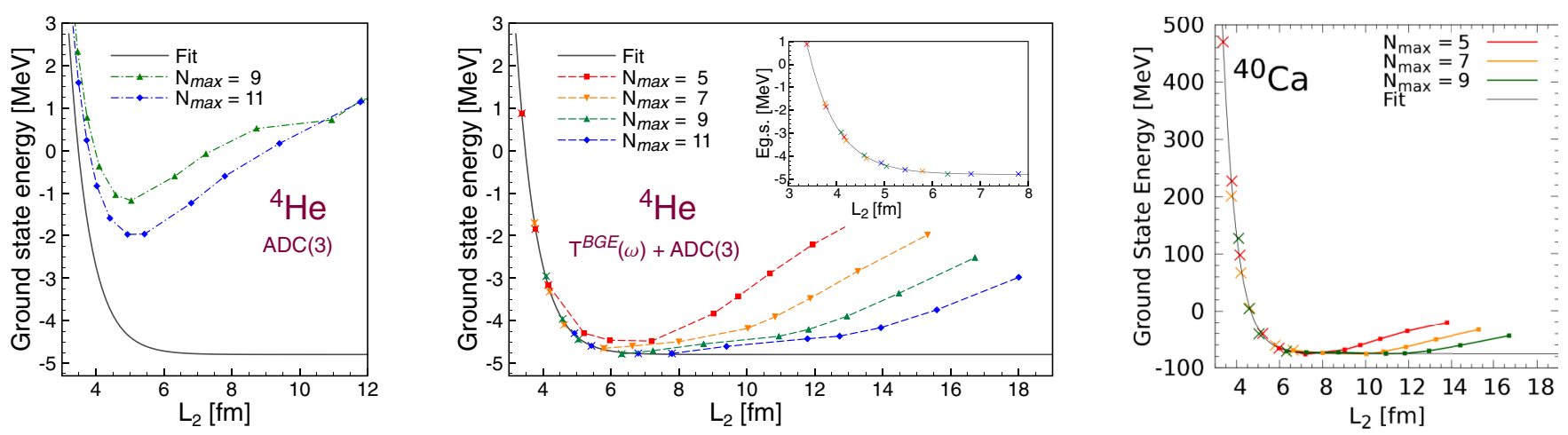

FIG. 3. Calculated ground-state energies of ${ }^{4} \mathrm{He}$ and ${ }^{40} \mathrm{Ca}$ for the HAL469 potential as a function of the effective box radius $L_{2}$. Left: Solution for the bare interaction at $N_{\max }=9$ and 11 and varying oscillator frequencies without ladders from the excluded space $Q$. Middle: Full calculation, including all ladder diagrams in $Q$. Different colors and broken lines are a guide to the eye connecting results of the same $N_{\max }$. The data points included in the fit are marked with crosses and are also shown separately in the inset. Right: Same as the middle panel but for ${ }^{40} \mathrm{Ca}$. For all panels, the full black line is the result of the IR extrapolation, with the inclusion of the $T^{\mathrm{BGE}}$ ladder, according to Eq. (7).

lines in Fig. 3 are the result of a nonlinear least-squares fit to Eq. (7). The points diverging from the exponential behavior at large $L_{2}$ are assumed not to be UV converged due to the above-mentioned approximations and are excluded from the fit but are still shown in the figure. From calculations up to $\hbar \Omega=50 \mathrm{MeV}$ and the IR extrapolation, we estimate a converged binding energy of 4.80 (3) $\mathrm{MeV}$ for ${ }^{4} \mathrm{He}$, where the error corresponds to the uncertainties in the extrapolation. The calculations for the other isotopes converge similarly to ${ }^{4} \mathrm{He}$, and we show the IR extrapolation of ${ }^{40} \mathrm{Ca}$ in the right panel of Fig. 3, for completeness. The figure is also indicative that this methodology can be successfully applied to heavier nuclei. These results clearly show that SCGF can handle relatively hard potentials by resumming of ladders through the Bethe-Goldstone equation and they give confidence that the short-range repulsion of HAL469 is accounted for accurately.

Results. The calculated ground-state energies of ${ }^{4} \mathrm{He},{ }^{16} \mathrm{O}$, and ${ }^{40} \mathrm{Ca}$ are summarized in Table I, together with BHF results obtained with the same gap choice and methods of Ref. [25]. For ${ }^{4} \mathrm{He}$, the complete $T^{\mathrm{BGE}}(\omega)+\mathrm{ADC}(3)$ result deviates from the exact solution for ${ }^{4} \mathrm{He}$ by less than $10 \%$. Since the SCGF approach resums linked diagrams, and thus it is size extensive, one should expect that similar errors will apply for larger isotopes [57]. Thus, Table I shows both the uncertainties in

TABLE I. Ground-state energies of ${ }^{4} \mathrm{He},{ }^{16} \mathrm{O}$, and ${ }^{40} \mathrm{Ca}$ at $M_{\mathrm{PS}}=469 \mathrm{MeV} / c^{2}$ obtained from the HAL469 interaction. The $T^{\mathrm{BGE}}(\omega)+\mathrm{ADC}(3)$ results of the present work are compared to $\mathrm{BHF}$ and to the exact solution. The last line is the breakup energy for splitting the system into ${ }^{4} \mathrm{He}$ clusters (of total energy $A / 4 \times 5.09 \mathrm{MeV}$ ).

\begin{tabular}{|c|c|c|c|}
\hline$E_{0}^{A}(\mathrm{MeV})$ & ${ }^{4} \mathrm{He}$ & ${ }^{16} \mathrm{O}$ & ${ }^{40} \mathrm{Ca}$ \\
\hline BHF [25] & -8.2 & -34.7 & -11 \\
\hline$T^{\mathrm{BGE}}(\omega)+\mathrm{ADC}(3)$ & $-4.80(0.03)$ & $-17.9(0.3)(1.8)$ & $-75.4(6.7)(7.5)$ \\
\hline Exact calc. [56] & -5.09 & & \\
\hline Experiment & -28.3 & -127.7 & -342.0 \\
\hline Separation into ${ }^{4} \mathrm{H}$ & le clusters: & $-2.46(0.3)(1.8)$ & $24.5(6.7)(7.5)$ \\
\hline
\end{tabular}

the IR extrapolation [55] and an error for the many-body truncations, for we which we take a conservative estimate of $10 \%$ based on our finding for ${ }^{4} \mathrm{He}$. The SCGF results are sensibly less bound than our previous BHF results [25] and we interpret this as a limitation of BHF theory.

A key feature of our calculations is the use of a harmonic oscillator space, which effectively confines all nucleons. The last line of Table I reports the deduced breakup energies for separating the computed ground states into infinitely distant ${ }^{4} \mathrm{He}$ clusters. The ${ }^{16} \mathrm{O}$ is unstable with respect to the $4-\alpha$ breakup, by $\approx 2.5 \mathrm{MeV}$. Allowing an error in our binding energies of more than $10 \%$ could make oxygen bound but only very weakly. This is in contrast to the experimental results, at the physical quarks masses, where the 4- $\alpha$ breakup requires 14.4 MeV. On the other hand, ${ }^{40} \mathrm{Ca}$ is stable with respect to breakup in $\alpha$ particles by $\approx 24 \mathrm{MeV}$. We expect that these observations are rather robust even when we consider the (LQCD) statistical errors in the HAL469 interaction. While such statistical fluctuations introduce additional $\sim 10 \%$ errors on binding energies [25], they are expected to be strongly correlated among ${ }^{4} \mathrm{He},{ }^{16} \mathrm{O}$, and ${ }^{40} \mathrm{Ca}$. Hence, for QCD in the $\mathrm{SU}(3)$ limit at $M_{\mathrm{PS}}=469 \mathrm{MeV} / c^{2}$, we find that the deuteron is unbound [23] and ${ }^{16} \mathrm{O}$ is only just slightly above the threshold for $\alpha$ breakup, while ${ }^{4} \mathrm{He}$ and ${ }^{40} \mathrm{Ca}$ are instead bound. The HAL469 interaction has the lowest $M_{\mathrm{PS}}$ value among those considered in Refs. [22,23], while from Ref. [24] we know that it is the only one saturating nuclear matter (although not at the physical saturation point). Moreover, we have tested that SCGF attempts at calculating asymmetric isotopes, like ${ }^{28} \mathrm{O}$, predict strongly unbound systems even for HAL469. All these results together suggest that when lowering of the pion mass toward its physical value, closed shell isotopes are created at first around the traditional magic numbers. This hypothesis should also be seen in light of the limitations in the present HAL469 Hamiltonian, which was built to include only the ${ }^{1} \mathrm{~S}_{0},{ }^{3} \mathrm{~S}_{1}$, and ${ }^{3} \mathrm{D}_{1}$ partial waves and therefore neglects the three-body forces and spin-orbit interactions. The missing $P$ waves and Coulomb force are repulsive but could be compensated for by an attracting three-body force. 


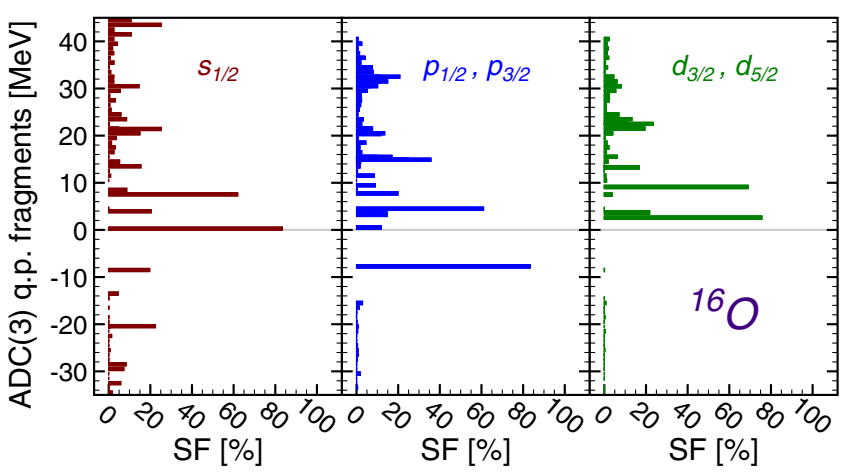

FIG. 4. Single-particle spectral strength distribution of ${ }^{16} \mathrm{O}$ obtained from the dressed propagator in the full $T^{\mathrm{BGE}}(\omega)$ plus $\mathrm{ADC}(3)$ approach. Each panel displays partial waves of different angular momenta. The vertical axes give the quasiparticle energies [that is, the poles of Eq. (1)], while the lengths of the horizontal bars give the calculated spectroscopic factors.

Figure 4 demonstrates the spectral strength distribution of ${ }^{16} \mathrm{O}$ obtained for $N_{\max }=11$ and $\hbar \Omega=11 \mathrm{MeV}$. Quasiparticle fragments corresponding to spin-orbit partners do not split due to the absence of a spin-orbit term in HAL469. Otherwise, all the remaining qualitative features of the experimental spectral distribution are seen also for the $M_{\mathrm{PS}}=469 \mathrm{MeV} / c^{2}$. The rms radii are given Table II for the same model space and oscillator frequency. Although the total binding energies are 15-20\% of the experimental value (Table I), the computed charge radii are about the same as the experiment. This is due to the fact that the heavy nucleon mass $\left(m_{N}=1161.1 \mathrm{MeV} / c^{2}\right)$ used here reduces the motion of the nucleons inside the nuclei and counterbalances the effect of weak attraction of the HAL469 potential. We have also checked the dependence of the computed radii on the effective model space size, Eq. (6), and found a rather flat converged region for all three isotopes, although the values still oscillate by about $0.05 \mathrm{fm}$ with changing $L_{2}$. The values in Table II are all calculated in the middle of this plateau. The HF approach of Eq. (4) and the standard BHF give similar radii even though they predict very different binding energies. The final radii are then increased by many-body correlations and, for all nuclei, the full $T^{\mathrm{BGE}}(\omega)$ plus $\mathrm{ADC}(3)$ calculation pushes the matter distribution to larger distances. However,

TABLE II. Computed matter and charge radii of ${ }^{16} \mathrm{O}$ and ${ }^{40} \mathrm{Ca}$ using $\mathrm{M}_{\mathrm{PS}}=469 \mathrm{MeV}$ for $N_{\max }=11$ and $\hbar \Omega=11 \mathrm{MeV}$. Results are given for different levels of approximation and the charge radii from the full $T^{\mathrm{BGE}}(\omega)$ plus $\mathrm{ADC}(3)$ are compared to the experimental values. For charge radii, we assumed the physical charge distributions of the protons and neutrons (see Ref. [60] for details).

\begin{tabular}{lcccc}
\hline \hline & & ${ }^{4} \mathrm{He}$ & ${ }^{16} \mathrm{O}$ & ${ }^{40} \mathrm{Ca}$ \\
\hline$r_{\text {pt-matter }}(\mathrm{fm}):$ & BHF $[25]$ & 2.09 & 2.35 & 2.78 \\
& $\mathrm{HF}$ & 1.62 & 2.39 & 2.78 \\
& $T^{\mathrm{BGE}}(\omega)+\mathrm{ADC}(3)$ & 1.67 & 2.64 & 2.97 \\
$r_{\text {charge }}(\mathrm{fm}):$ & $T^{\mathrm{BGE}}(\omega)+\mathrm{ADC}(3)$ & 1.89 & 2.79 & 3.10 \\
& Experiment $[58,59]$ & 1.67 & 2.73 & 3.48 \\
\hline \hline
\end{tabular}

we note that accounting for the neglected high-momentum components-as discussed below Eq. (5)—-tends to enhance the central density and would slightly reduce the calculated radii [61].

Summary. We investigated the use of the BGE in an ab initio approach and used it to resum missing two-nucleon scattering diagrams outside the usual truncations of the many-body space, while the full $\mathrm{ADC}(3)$ method has been retained within the model space itself. A benchmark on ${ }^{4} \mathrm{He}$ shows that the present implementation works relatively well and it allows us to solve the self-consistent Green's function for the HAL QCD potentials derived from lattice QCD. An investigation of the IR convergence of the ground-state energies, following the work of Ref. [55], indicates that SCGF can handle relatively hard potentials such as the HAL469, even for masses as large as $A=40$. This opens a new path that allows full $a b$ initio calculations of large nuclei even with hard nuclear interactions.

The present accuracy is sufficient to make quantitative statements on doubly magic nuclei, which are less bound compared to earlier BHF estimates for the HAL QCD potentials. Here, we have found that the behavior when lowering the pion mass toward its physical value is consistent with the idea that nuclei near the traditional magic numbers are formed at first. At $M_{\mathrm{PS}}=469 \mathrm{MeV} / c^{2}$, in the SU(3) limit of QCD, both ${ }^{4} \mathrm{He}$ and ${ }^{40} \mathrm{Ca}$ have bound ground states while the deuteron is unbound and ${ }^{16} \mathrm{O}$ is likely to decay into four separate $\alpha$ particles. However, ${ }^{16} \mathrm{O}$ is already close to becoming bound. This suggests that the region of $M_{\mathrm{PS}} \sim 500 \mathrm{MeV} / c^{2}$ marks a transition between an unbound nuclear chart and the emergence of bound isotopes. Further studies at lower pion masses will be pivotal to testing these findings and should be possible in the near future since LQCD simulations for nuclear and hyperon forces down to physical quark masses are currently underway [39-41].

Important future work will also be the inclusion of the spinorbit as well as three-nucleon forces. Proof-of-principle LQCD calculations for these interactions are available $[14,62]$ and follow the hierarchy of nuclear forces with three-nucleon terms smaller than the $N N$ contributions.

Acknowledgments. We thank the HAL QCD Collaboration for providing the HAL469 $9_{\mathrm{SU}(3)}$ interaction. Computations of the $T^{\mathrm{BGE}}(\omega)$ were performed using the CENS codes from Ref. [51]. This work was supported in part by the United Kingdom Science and Technology Facilities Council (STFC) under Grants No. ST/L005743/1 and No. ST/L005816/1, by the Royal Society International Exchanges Grant No. IE150305, by Japanese Grant-in-Aid for Scientific Research [JP15K17667and (C)26400281], by MEXT Strategic Program for Innovative Research (SPIRE) Field 5, by a priority issue (Elucidation of the fundamental laws and evolution of the universe) to be tackled by using the Post " $\mathrm{K}$ " Computer, and by the Joint Institute for Computational Fundamental Science (JICFuS). T.D and T.H. were supported partially by the RIKEN iTHEMS Program and iTHES Project. Calculations were performed at the DiRAC Complexity system at the University of Leicester (funded by Department for Business Innovation and Skills (BIS) National E-infrastructure capital Grant No. ST/K000373/1 and STFC Grant No. ST/K0003259/1). 
[1] S. Borsanyi, S. Durr, Z. Fodor, C. Hoelbling, S. D. Katz, S. Krieg, L. Lellouch, T. Lippert, A. Portelli, K. K. Szabo, and B. C. Toth, Science 347, 1452 (2015).

[2] S. R. Beane, E. Chang, W. Detmold, H. W. Lin, T. C. Luu, K. Orginos, A. Parreño, M. J. Savage, A. Torok, and A. WalkerLoud (NPLQCD Collaboration), Phys. Rev. D 85, 054511 (2012).

[3] T. Yamazaki, K.-i. Ishikawa, Y. Kuramashi, and A. Ukawa, Phys. Rev. D 86, 074514 (2012).

[4] T. Yamazaki, K.-i. Ishikawa, Y. Kuramashi, and A. Ukawa, Phys. Rev. D 92, 014501 (2015).

[5] K. Orginos, A. Parreño, M. J. Savage, S. R. Beane, E. Chang, and W. Detmold (NPLQCD Collaboration), Phys. Rev. D 92, 114512 (2015).

[6] E. Berkowitz, T. Kurth, A. Nicholson, B. Joo, E. Rinaldi, M. Strother, P. M. Vranas, and A. Walker-Loud, Phys. Lett. B 765, 285 (2017).

[7] T. Iritani, T. Doi, S. Aoki, S. Gongyo, T. Hatsuda, Y. Ikeda, T. Inoue, N. Ishii, K. Murano, H. Nemura, and K. Sasaki, J. High Energy Phys. 10 (2016) 101.

[8] S. Aoki, T. Doi, and T. Iritani, PoS(LATTICE2016), 109 (2016).

[9] T. Iritani, PoS(LATTICE2016), 107 (2016).

[10] N. Ishii, S. Aoki, and T. Hatsuda, Phys. Rev. Lett. 99, 022001 (2007).

[11] S. Aoki, T. Hatsuda, and N. Ishii, Prog. Theor. Phys. 123, 89 (2010).

[12] S. Aoki, T. Doi, T. Hatsuda, Y. Ikeda, T. Inoue, N. Ishii, K. Murano, H. Nemura, and K. Sasaki (HAL QCD Collaboration), Prog. Theor. Exp. Phys. 2012, $01 \mathrm{~A} 105$ (2012).

[13] S. Aoki, N. Ishii, T. Doi, Y. Ikeda, and T. Inoue, Phys. Rev. D 88, 014036 (2013).

[14] T. Doi, S. Aoki, T. Hatsuda, Y. Ikeda, T. Inoue, N. Ishii, K. Murano, H. Nemura, and K. Sasaki (HAL QCD Collaboration), Prog. Theor. Phys. 127, 723 (2012).

[15] N. Ishii, S. Aoki, T. Doi, T. Hatsuda, Y. Ikeda, T. Inoue, K. Murano, H. Nemura, and K. Sasaki, Phys. Lett. B 712, 437 (2012).

[16] T. Doi and M. G. Endres, Comput. Phys. Commun. 184, 117 (2013).

[17] W. Detmold and K. Orginos, Phys. Rev. D 87, 114512 (2013).

[18] J. Günther, B. C. Tóth, and L. Varnhorst, Phys. Rev. D 87, 094513 (2013).

[19] H. Nemura, Comput. Phys. Commun. 207, 91 (2016).

[20] N. Barnea, L. Contessi, D. Gazit, F. Pederiva, and U. van Kolck, Phys. Rev. Lett. 114, 052501 (2015).

[21] L. Contessi, A. Lovato, F. Pederiva, A. Roggero, J. Kirscher, and U. van Kolck, Phys. Lett. B 772, 839 (2017).

[22] T. Inoue, N. Ishii, S. Aoki, T. Doi, T. Hatsuda, Y. Ikeda, K. Murano, H. Nemura, and K. Sasaki (HAL QCD Collaboration), Phys. Rev. Lett. 106, 162002 (2011).

[23] T. Inoue, S. Aoki, T. Doi, T. Hatsuda, Y. Ikeda, N. Ishii, K. Murano, H. Nemura, and K. Sasaki, Nucl. Phys. A 881, 28 (2012).

[24] T. Inoue, S. Aoki, T. Doi, T. Hatsuda, Y. Ikeda, N. Ishii, K. Murano, H. Nemura, and K. Sasaki (HAL QCD Collaboration), Phys. Rev. Lett. 111, 112503 (2013).

[25] T. Inoue, S. Aoki, B. Charron, T. Doi, T. Hatsuda, Y. Ikeda, N. Ishii, K. Murano, H. Nemura, and K. Sasaki (HAL QCD Collaboration), Phys. Rev. C 91, 011001(R) (2015).

[26] V. Somà, A. Cipollone, C. Barbieri, P. Navrátil, and T. Duguet, Phys. Rev. C 89, 061301 (2014).
[27] H. Hergert, S. K. Bogner, T. D. Morris, S. Binder, A. Calci, J. Langhammer, and R. Roth, Phys. Rev. C 90, 041302 (2014).

[28] G. Hagen, T. Papenbrock, M. Hjorth-Jensen, and D. J. Dean, Rep. Prog. Phys. 77, 096302 (2014).

[29] W. Dickhoff and C. Barbieri, Prog. Part. Nucl. Phys. 52, 377 (2004).

[30] A. Carbone, A. Cipollone, C. Barbieri, A. Rios, and A. Polls, Phys. Rev. C 88, 054326 (2013).

[31] K. Hebeler, J. Holt, J. Menéndez, and A. Schwenk, Annu. Rev. Nucl. Part. Sci. 65, 457 (2015).

[32] A. Cipollone, C. Barbieri, and P. Navrátil, Phys. Rev. Lett. 111, 062501 (2013).

[33] A. Ekström, G. R. Jansen, K. A. Wendt, G. Hagen, T. Papenbrock, B. D. Carlsson, C. Forssén, M. Hjorth-Jensen, P. Navrátil, and W. Nazarewicz, Phys. Rev. C 91, 051301 (2015).

[34] V. Lapoux, V. Somà, C. Barbieri, H. Hergert, J. D. Holt, and S. R. Stroberg, Phys. Rev. Lett. 117, 052501 (2016).

[35] R. F. Garcia Ruiz, M. L. Bissell, K. Blaum, A. Ekstrom, N. Frommgen, G. Hagen, M. Hammen, K. Hebeler, J. D. Holt, G. R. Jansen, M. Kowalska, K. Kreim, W. Nazarewicz, R. Neugart, G. Neyens, W. Nortershauser, T. Papenbrock, J. Papuga, A. Schwenk, J. Simonis, K. A. Wendt, and D. T. Yordanov, Nat. Phys. 12, 594 (2016).

[36] C. Barbieri and W. H. Dickhoff, Phys. Rev. C 65, 064313 (2002).

[37] C. Barbieri, Phys. Lett. B 643, 268 (2006).

[38] C. Barbieri and M. Hjorth-Jensen, Phys. Rev. C 79, 064313 (2009).

[39] T. Doi et al., in Proceedings, 33rd International Symposium on Lattice Field Theory (Lattice 2015): Kobe, Japan, July 14-18, 2015, PoS(LATTICE 2015), 086 (2015).

[40] N. Ishii et al., Proceedings, 33rd International Symposium on Lattice Field Theory (Lattice 2015): Kobe, Japan, July 14-18, 2015, PoS(LATTICE 2015), 087 (2015).

[41] K. Sasaki et al., Proceedings, 33rd International Symposium on Lattice Field Theory (Lattice 2015): Kobe, Japan, July 14-18, 2015, PoS(LATTICE 2015), 088 (2015).

[42] H. Nemura et al., JPS Conf. Proc. 17, 052002 (2017).

[43] A. Carbone (private communication).

[44] C. Barbieri and A. Carbone, in An Advanced Course in Computational Nuclear Physics: Bridging the Scales from Quarks to Neutron Stars, Lecture Notes in Physics Vol. 936, edited by M. Hjorth-Jensen, M. P. Lombardo, and U. van Kolck (Springer International, Berlin, 2017) Chap. 11, pp. 571-644.

[45] A. Fetter and J. Walecka, Quantum Theory of Many-Particle Systems (Dover, New York, 2003).

[46] W. H. Dickhoff and D. Van Neck, Many-Body Theory Exposed!, 2nd ed. (World Scientific, Singapore, 2008).

[47] J. Schirmer, L. S. Cederbaum, and O. Walter, Phys. Rev. A 28, 1237 (1983).

[48] C. Barbieri, D. Van Neck, and W. H. Dickhoff, Phys. Rev. A 76, 052503 (2007).

[49] V. Somà, C. Barbieri, and T. Duguet, Phys. Rev. C 89, 024323 (2014).

[50] M. Hjorth-Jensen, T. T. Kuo, and E. Osnes, Phys. Rep. 261, 125 (1995).

[51] T. Engeland, M. Hjorth-Jensen, and G. Jansen, CENS, a Computational Environment for Nuclear Structure, https://github.com/ManyBodyPhysics/CENS/tree/master/ $\mathrm{MBPT} /$.

[52] K. Gad and H. Müther, Phys. Rev. C 66, 044301 (2002). 
[53] T. C. Luu, S. Bogner, W. C. Haxton, and P. Navrátil, Phys. Rev. C 70, 014316 (2004).

[54] H. Hergert and R. Roth, Phys. Lett. B 682, 27 (2009).

[55] S. N. More, A. Ekström, R. J. Furnstahl, G. Hagen, and T. Papenbrock, Phys. Rev. C 87, 044326 (2013).

[56] H. Nemura, Int. J. Mod. Phys. E 23, 1461006 (2014).

[57] I. Shavitt and R. Bartlett, Many-Body Methods in Chemistry and Physics: MBPT and Coupled-Cluster Theory, Cambridge Molecular Science (Cambridge University, Cambridge, England, 2009).
[58] H. D. Vries, C. D. Jager, and C. D. Vries, At. Data Nucl. Data Tables 36, 495 (1987).

[59] I. Angeli and K. Marinova, At. Data Nucl. Data Tables 99, 69 (2013).

[60] A. Cipollone, C. Barbieri, and P. Navrátil, Phys. Rev. C 92, 014306 (2015).

[61] H. Müther and I. Sick, Phys. Rev. C 70, 041301 (2004).

[62] K. Murano, N. Ishii, S. Aoki, T. Doi, T. Hatsuda, Y. Ikeda, T. Inoue, H. Nemura, and K. Sasaki, Phys. Lett. B 735, 19 (2014). 